\title{
LAMB WAVE MICROMECHANICAL RESONATORS FORMED IN THIN PLATES OF LITHIUM NIOBATE
}

\author{
R.H. Olsson III, K. Hattar, M.S. Baker, M. Wiwi, J. Nguyen, C. Padilla, S.J. Homeijer, J. R. Wendt and \\ T.A. Friedmann \\ Sandia National Laboratories, Albuquerque, New Mexico, USA
}

\begin{abstract}
We study and compare high coupling symmetric and shear mode Lamb wave resonators realized in thin plates of X-cut lithium niobate. Fundamental mode bar resonators with a plate width of $20 \mu \mathrm{m}$, a plate thickness of $1.5 \mu \mathrm{m}$, apertures of 50,90 and $130 \mu \mathrm{m}$ and acoustic wave propagation rotated $30^{\circ}$ (symmetric) and $170^{\circ}$ (shear) to the +y-axis were realized on a single die for direct comparison. As expected, the symmetric Lamb wave resonators exhibited a higher sound velocity of $\sim 6400 \mathrm{~m} / \mathrm{s}$ when compared to the shear velocity of $\sim 3900 \mathrm{~m} / \mathrm{s}$. The shear mode resonators, however, were found to have a significantly higher effective piezoelectric coupling coefficient of $16.3 \%$, compared to a maximum of $9.1 \%$ for the symmetric Lamb wave resonators. In addition, the shear mode resonators were found to be less sensitive to the device aperture and to have fewer spurious responses. Based on these results, the shear mode resonators were selected for scaling to higher operating frequencies. A shear mode lithium niobate Lamb wave resonator operating at $350 \mathrm{MHz}$ has been demonstrated with an effective piezoelectric coupling of $16 \%$, a quality factor in air of 2200 and a device figure-of-merit of 420 , among the highest reported for Lamb wave resonators [1-3].
\end{abstract}

\section{INTRODUCTION}

Microresonator filter arrays have been studied as a smaller, more highly integrated replacement for the numerous filters dies that currently reside in the RF front-end of a multi-band cellular handset. In particular, microresonators realized in thin films of lithium niobate $\left(\mathrm{LiNbO}_{3}\right)$ [1-3] have demonstrated the high piezoelectric coupling needed to realize band select filters with percent bandwidths of $2-5 \%$, while simultaneously exhibiting the high quality factors required for duplexers with narrow frequency gaps between the transmit and receive bands.

In this paper we study and compare the properties of Lamb wave resonators vibrating in the fundamental symmetric (S0) and shear (SH0) modes. These modes were chosen because they are predicted [4] to have both low dispersion and high coupling coefficient over a wide range of thickness-to-wavelength ratios $(h / \lambda)$. Both of these properties are important for realizing multifrequency band select filters in a single $\mathrm{LiNbO}_{3}$ layer.

Fundamental mode resonators with a plate width of $20 \mu \mathrm{m}$, a plate thickness of $1.5 \mu \mathrm{m}$, varying apertures and acoustic wave propagation rotated $30^{\circ}(\mathrm{S} 0)$ and $170^{\circ}(\mathrm{SH} 0)$ to the $+\mathrm{y}$-axis to maximize piezoelectric coupling [4] were realized on a single die for direct comparison. The $h / \lambda$ of 0.04 is very close to the optimum value to maximize piezoelectric coupling of 0.05 found for both the SO and SH0 modes in [4]. We find that while the S0 Lamb wave has a 1.6 times higher sound velocity than the SH0 mode, the SH0 mode is predicted to and consistently exhibits a 1.6-1.8 times higher effective piezoelectric coupling coefficient, $k_{e f f}^{2}$. The SH0 mode also exhibits higher quality factor $(Q)$, higher figure-of-merit $(M)$ and fewer spurious responses.

Finally, a fundamental SH0 mode Lamb wave resonator realized in a $4.4 \mu \mathrm{m}$ wide plate is demonstrated with an operating frequency of $350 \mathrm{MHz}$, a $k_{\text {eff }}^{2}=16 \%$, a $Q=2200$ in air and a $M=$ 420 , among the highest reported for Lamb wave resonators.

\section{DEVICE FABRICATION}

The resonators are fabricated using the process flow shown in Fig. 1 [3], where selective ion irradiation of a $\mathrm{LiNbO}_{3}$ wafer creates a damaged release layer that selectively etches in a HF chemistry, allowing suspended membranes to be formed. The advantages of this fabrication process when compared to prior art [1,2] are: 1) the ability to lithographically define the undercut of the device, 2) no wafer bonding, polishing or fracturing and 3) the ability to realize custom and potentially multiple $\mathrm{LiNbO}_{3}$ thicknesses on a single substrate.

The process begins with an $\mathrm{X}$-cut $\mathrm{LiNbO}_{3}$ wafer upon which a $100 \mathrm{~nm}$ layer of $\mathrm{Cr}$ is deposited and patterned to form alignment marks. Next, a $\mathrm{SiO}_{2}$ layer is deposited and patterned to determine where the release layer will be formed via ion irradiation. The sample is then implanted with a He dose of $1 \times 10^{16}$ atoms $/ \mathrm{cm}^{3}$ at an energy of $0.9 \mathrm{MeV}$ to create an ion damaged release layer of $\mathrm{LiNbO}_{3}$ approximately $2 \mu \mathrm{m}$ below the wafer surface. This energy was found to give a final plate thickness after release of approximately $1.5 \mu \mathrm{m}$. The implants are performed in a $3 \mathrm{MV}$ NEC Pelletron using a current of $<7 \mu \mathrm{A}$. A low sample temperature is maintained via liquid nitrogen cooling using a $\mathrm{Cu}$ braid during implantation. The ion implant is intentionally performed through the $\mathrm{Cr}$ electrode layer to promote adhesion. After ion implantation, the $\mathrm{SiO}_{2}$ implant masking layer is stripped and the $\mathrm{LiNbO}_{3}$ is patterned using an oxide hard mask and $\mathrm{Cl}$ dry etching to define the final plate dimensions. Next, the $\mathrm{Cr}$ electrodes are patterned and a layer of $\mathrm{Au}$ is deposited and patterned via lift off to protect the $-\mathrm{z}$ face of the $\mathrm{LiNbO}_{3}$ device, which can have a significant etch rate in HF during the release. During our research we found that the structural rigidity of this $\mathrm{Au}$ protection layer also led to significantly improved device yield through the release process. Finally the devices are released in a $\mathrm{HF}$ based chemistry and the Au protect layer is stripped in $\mathrm{KI}-\mathrm{I}_{2}$.

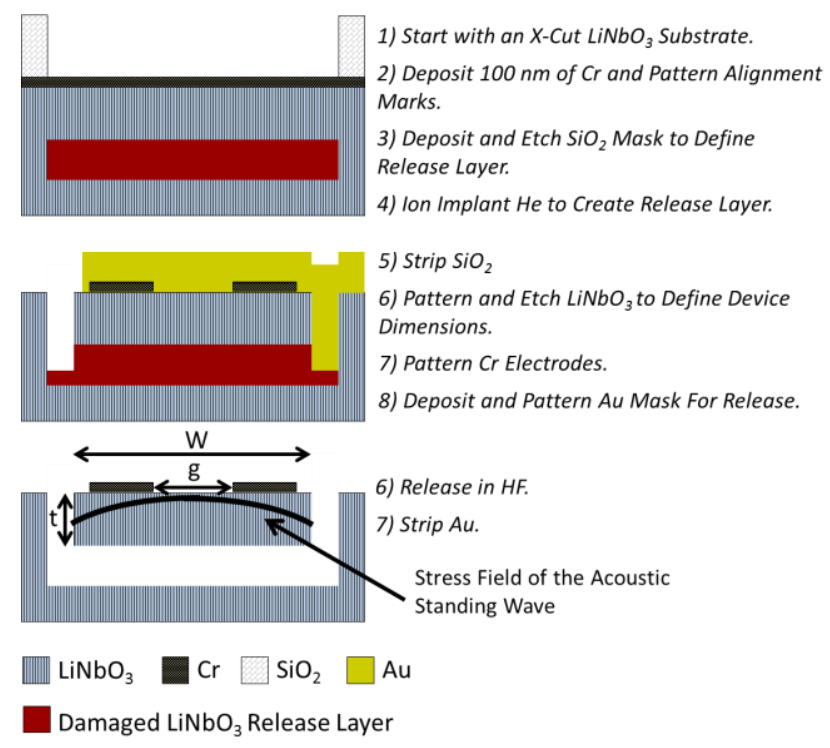

Figure 1: $\mathrm{LiNbO}_{3}$ microresonator fabrication process.

Solid-State Sensors, Actuators and Microsystems Workshop Hilton Head Island, South Carolina, June 8-12, 2014 


\section{DEVICE STRUCTURE AND DIMENSIONS}

A final cross-section schematic of the resonator is shown in the bottom of Fig. 1 and a scanning electron microscope (SEM) image of one of the resonators studied in this work is shown in Fig. 2. The resonator consists of a $1.5 \mu \mathrm{m}$ thick suspended membrane of $\mathrm{LiNbO}_{3}$ with a width, $W=20 \mu \mathrm{m}$. The device was designed to resonate in the fundamental S0 or SH0 mode, see Fig. 3, with a resonant frequency, $f_{s}=c / 2 \mathrm{~W}$, where $c$ is the sound velocity of the Lamb wave. The thickness-to-wavelength ratio, $t / \lambda=0.04$, is closer to the optimum value to maximize $k_{\text {eff }}^{2}$ of 0.05 [4] than previous work [3]. The space between the electrodes and device substrate anchor, $s$, was $45 \mu \mathrm{m}$ for all devices. While it was found in $[2,3]$ that to maximize $k_{\text {eff }}$ the electrodes should be placed at the edges of the resonator, $5 \mu \mathrm{m}$ wide electrodes with a gap, $g=5 \mu \mathrm{m}$, were designed slightly offset from the resonator edge for ease of fabrication. Electrode apertures, $a$, which define the resonator static capacitance, of 50,90 and $130 \mu \mathrm{m}$ were modeled, fabricated and characterized.

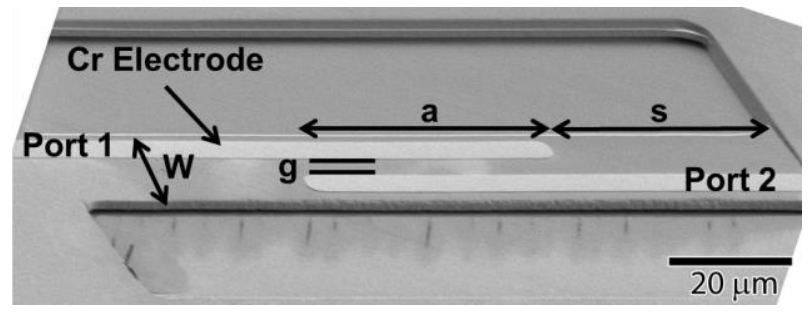

Figure 2: $\mathrm{LiNbO}_{3}$ microresonator SEM image.

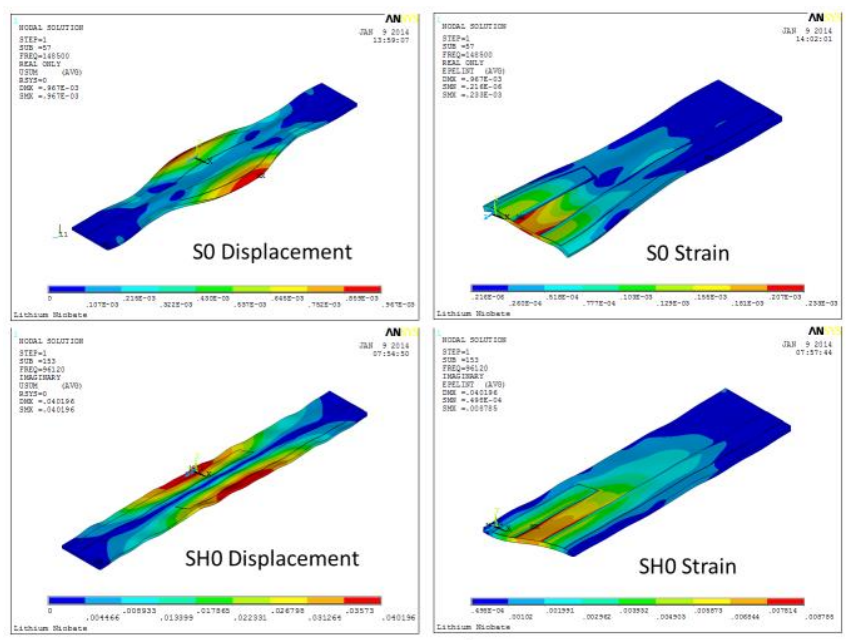

Figure 3: Displacement (Full) and strain (Half) profiles of the SO and $\mathrm{SHO}$ resonator modes with an aperture of $50 \mu \mathrm{m}$.

\section{FINITE ELEMENT MODELING RESULTS}

The six devices with three different apertures and two different rotations to the +y-axis described above were studied using finite element modeling (FEM) and experimentally. The FEM was a full 3D representation of the device including the anchors and electrodes. Figure 3 shows the displacement and strain profiles from ANSYS FEM for the S0 Lamb wave resonator rotated $30^{\circ}$ to the $+\mathrm{y}$-axis and for the $\mathrm{SH} 0 \mathrm{Lamb}$ wave resonator rotated $170^{\circ}$ (SH0) to the +y-axis, both with apertures of $50 \mu \mathrm{m}$. The maximum strain is seen to appear between the $\mathrm{Cr}$ electrodes and is thus efficiently transduced by the electric field applied across these same electrodes. The strain between the electrodes at the edges of the aperture for the S0 Lamb wave resonator is seen to dramatically decrease, indicating that the S0 Lamb wave $k_{\text {eff }}^{2}$ will be significantly impacted by the device aperture. The $k_{\text {eff }}^{2}$ for each device was studied using harmonic analysis in ANSYS. The piezoelectric coupling, $K^{2}$, was calculated from the simulated resonator admittance using eq. 1 ,

$$
K^{2}=\frac{1}{2 \pi f_{s} Q R_{x} C_{S}},
$$

where $f_{s}$ is the frequency of minimum resonator admittance, $R_{X}$ is the resonator motional impedance, $C_{s}$, is the resonator static capacitance and $Q$ is the resonator quality factor which is an input to the FEM. The effective piezoelectric coupling was then calculated using eq. 2 ,

$$
k_{e f f}^{2}=\frac{K^{2}}{1+K^{2}} .
$$

In our previous work [3] $k_{\text {eff }}^{2}$ was calculated from the simulated resonator admittance using eq. 3 ,

$$
k_{e f f}^{2}=\frac{f_{p}^{2}-f_{s}^{2}}{f_{p}^{2}},
$$

where $f_{p}$ is the frequency of maximum resonator admittance. The results of equations 2 and 3 are equal when no spurious resonances are seen near $f_{s}$ or $f_{p}$. In this work, however, we find in both FEM and in experiments that spurious modes cause a significant increase in $f_{p}$ and overestimation of $k_{\text {eff }}^{2}$ if equation 3 is used. We note here that the piezoelectric coupling coefficient, $k_{t}^{2}$, reported in [1] is equal to,

$$
k_{t}^{2}=\frac{\pi^{2}}{8} \frac{C_{X}}{C_{S}}=\frac{\pi^{2}}{8} K^{2}=\frac{\pi^{2}}{8} \frac{k_{e f f^{2}}}{1-k_{e f f}{ }^{2}} .
$$

The simulated $k_{\text {eff }}^{2}$ vs. aperture is shown in Fig. 4 and summarized in Table 1 for both the SO and SH0 Lamb wave resonators. The S0 Lamb wave resonator is predicted to have a significantly lower $k_{e f f}^{2}$ that is much more sensitive to the aperture than the SH0 Lamb wave resonator.

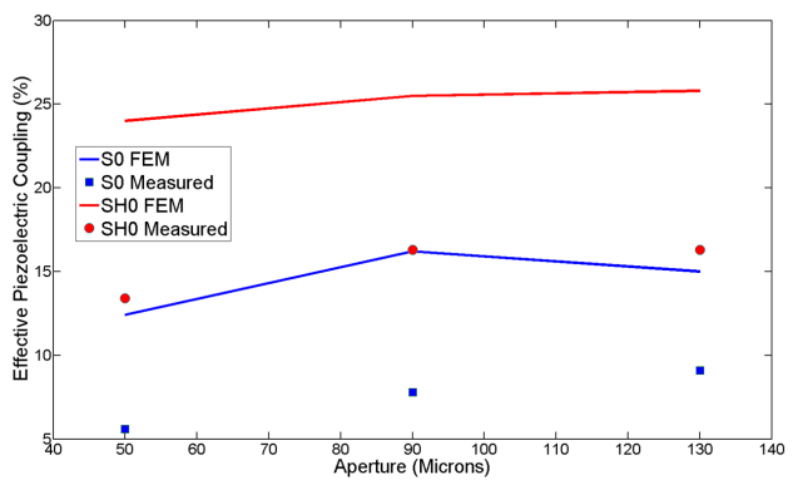

Figure 4: FEM and experimental $k_{\text {eff }}^{2}$ vs. aperture.

\section{EXPERIMENTAL RESULTS}

The admittance of the six different resonators realized on the same die was measured in air using a network analyzer. The responses for the $\mathrm{S} 0$ and $\mathrm{SH} 0$ mode resonators with an aperture of $90 \mu \mathrm{m}$ are shown in Fig. 5 (S0) and in Fig. 6 (SH0). Also shown in Fig. 5 and Fig. 6 are the results of the FEM for each device simulated with the measured quality factors. Each resonator measurement was fit to the modified Butterworth Van Dyke (MBVD) equivalent circuit model shown in Fig. 7 using eq. 5-7, 
Table 1. Experimental and FEM results for the SO and SHO Lamb wave micromechanical resonators.

\begin{tabular}{|c|c|c|c|c|c|c|c|c|c|c|c|c|}
\hline Mode & $\begin{array}{c}\text { Orientation } \\
\text { to }+Y \text { axis }\end{array}$ & $\begin{array}{c}\text { Aperture } \\
(\mu \mathrm{m})\end{array}$ & $\begin{array}{l}\text { Freq. } \\
\text { (MHz) }\end{array}$ & $\begin{array}{l}\mathbf{R}_{\mathrm{S}} \\
(\mathbf{\Omega})\end{array}$ & $\begin{array}{l}\mathbf{R}_{\mathbf{X}} \\
(\mathbf{\Omega})\end{array}$ & $\begin{array}{c}\mathbf{C}_{S} \\
(\mathbf{f F})\end{array}$ & $\begin{array}{c}\text { FEM } \\
{\text { Model } k_{\text {eff }}^{2}}_{(\%)}\end{array}$ & $\begin{array}{c}\text { Measured } \\
k_{\text {eff }}^{2}[2,3] \\
(\%)\end{array}$ & $\begin{array}{l}\text { Measured } \\
\mathbf{k}_{\mathbf{t}}^{2}[1](\%)\end{array}$ & $\mathbf{Q}_{\text {Total }}$ & $\mathbf{Q}_{\mathrm{A}}$ & $\begin{array}{c}\text { FOM } \\
\mathbf{k}_{\mathrm{eff}}^{2} \mathbf{Q}_{\mathrm{A}} /\left(1-\mathrm{k}_{\mathrm{eff}}{ }^{2}\right)\end{array}$ \\
\hline S0 & $30^{\circ}$ & 50 & 158.9 & 194 & 3516 & 9 & 12.4 & 5.6 & 7.4 & 500 & 528 & 31 \\
\hline S0 & $30^{\circ}$ & 90 & 160.6 & 233 & 1386 & 12 & 16.2 & 7.8 & 10.5 & 600 & 701 & 59 \\
\hline S0 & $30^{\circ}$ & 130 & 161.5 & 272 & 944 & 18 & 15.0 & 9.1 & 12.3 & 450 & 580 & 58 \\
\hline SH0 & $170^{\circ}$ & 50 & 98.4 & 194 & 922 & 8.5 & 24.0 & 13.4 & 19.1 & 1100 & 1331 & 206 \\
\hline SH0 & $170^{\circ}$ & 90 & 96.9 & 233 & 702 & 12 & 25.5 & 16.3 & 24.1 & 750 & 999 & 195 \\
\hline SH0 & $170^{\circ}$ & 130 & 94.8 & 272 & 783 & 17 & 25.8 & 16.3 & 24.1 & 480 & 647 & 126 \\
\hline
\end{tabular}

$$
\begin{gathered}
R_{X}=\frac{1}{2 \pi f_{S} C_{S} K^{2} Q_{A}}, \\
C_{X}=\frac{C_{S}}{K^{2}}, \\
L_{X}=\frac{R_{x} Q_{A}}{2 \pi f_{S}}=\frac{\left(R_{x}+R_{S}\right) Q_{T o t a l}}{2 \pi f_{S}},
\end{gathered}
$$

where $C_{X}$ and $L_{X}$ are the motional capacitance and inductance, $R_{\mathrm{S}}$ is the series electrical resistance, $Q_{A}$ is the acoustic quality factor and $Q_{\text {Total }}$ is the measured $3 \mathrm{~dB}$ bandwidth of the acoustic admittance divided by $f_{s}$, which includes the losses from both $R_{X}$ and $R_{S}$. $\quad R_{S}$ was measured directly on a separate test structure allowing it to be extracted from $R_{X}$. The simulated response of the MBVD electrical equivalent circuit model for the S0 and SHO mode micromechanical resonators with an aperture of $90 \mu \mathrm{m}$ are shown in Fig. 5 and Fig. 6 along with the equivalent circuit parameters.

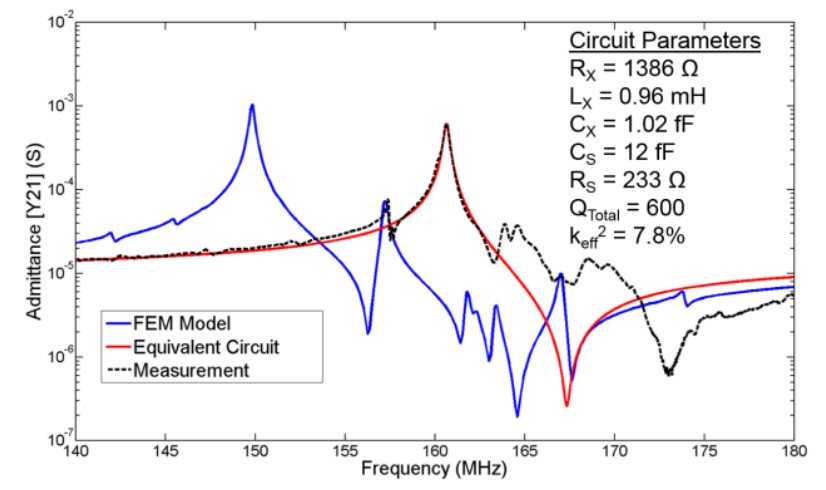

Figure 5: FEM and experimental results for the SO Lamb wave resonator with an aperture of $90 \mu \mathrm{m}$.

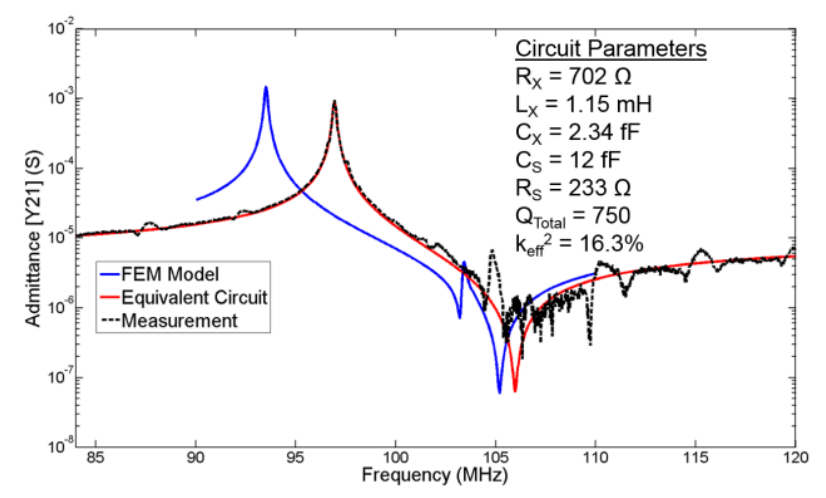

Figure 6: FEM and experimental results for the SHO Lamb wave resonator with an aperture of $90 \mu \mathrm{m}$.

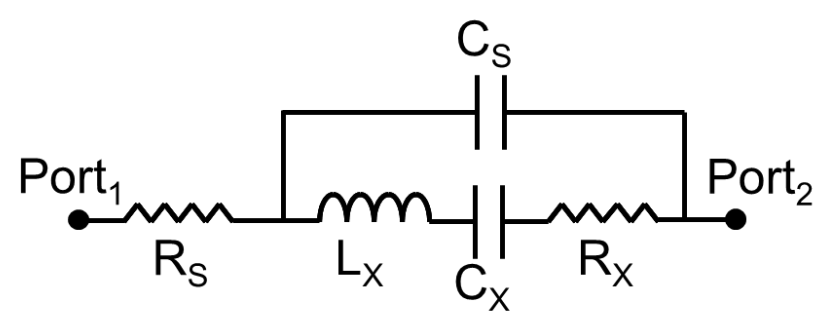

Figure 7: Modified BVD resonator equivalent circuit model.

The simulated MBVD results and experimental measurements are in good agreement far from resonance and close to the series resonance. The experimental $k_{\text {eff }}^{2}$ for each resonator was calculated from the MBVD equivalent circuit model using either equation 2 or 3 which yield identical results since no spurious responses are modeled using the circuit in Fig. 7. From Fig. 5 it is apparent that using the measured frequency of maximum admittance in equation 3 dramatically overestimates the $k_{\text {eff }}^{2}$ due to the spurious modes between $f_{s}$ and $f_{p}$. The measured $f_{s}, R_{S}, R_{X}, C_{S}$, $k_{e f f}^{2}, k_{t}^{2}, Q_{\text {Total }}, Q_{A}$ and acoustic figure-of-merit are summarized for all the resonators in Table 1 , while the measured $k_{\text {eff }}^{2}$ vs. aperture is shown in Fig. 4. The SH0 resonators are found to have significantly higher effective piezoelectric coupling coefficient and figure-of-merit.

\section{SCALING TO HIGHER FREQUENCIES}

From Table 1, the resistance of the $\mathrm{Cr}$ electrodes is seen to significantly degrade the total resonator quality factor. Higher frequency operation can only result in further degradation in $\mathrm{Q}_{\text {Total }}$ as the electrode cross sectional area will decrease, causing $R_{S}$ to increase and $R_{X}$ to decrease (see eq. 5). For this reason, when scaling to higher operating frequencies, the $\mathrm{Cr}$ electrodes in Fig. 1 were replaced with $100 \mathrm{~nm}$ of $\mathrm{Au}$ (lower resistivity compared to $\mathrm{Cr}$ ) and the $\mathrm{Au}-\mathrm{z}$ face protection mask step was omitted from the fabrication process.

The measured admittance of a SHO Lamb wave resonator rotated $170^{\circ}$ to the +y-axis with a plate width, $W=4.4 \mu \mathrm{m}$, an aperture, $a=60 \mu \mathrm{m}$, an electrode width of $1 \mu \mathrm{m}$ and a gap between the electrodes, $g=2 \mu \mathrm{m}$, is shown in Fig. 8 and Fig. 9. Also shown in both figures is the simulated response of the MBVD equivalent circuit. The narrowing of the plate width to $4.4 \mu \mathrm{m}$ has increased the series resonant frequency to $350 \mathrm{MHz}$. The inclusion of the $\mathrm{Au}$ electrodes has reduced the series electrical resistance to the point where it need not be modeled, $R_{S}=0 \Omega$ and $Q_{A}=Q_{\text {Total }}$. The increase in the effective electrode aperture to nearly 7 wavelengths has enabled a corresponding increase in the device quality factor to 2200. The ultra-high frequency (UHF) band resonator maintains a high effective coupling coefficient, $k_{e f f}^{2}=16 \%$ and a device figure-of-merit, $M=420$. The performance of the UHF, fundamental $\mathrm{SHO}$ mode $\mathrm{LiNbO}_{3}$ micromechanical resonator is summarized in Table 2. 
Table 2. Experimental results for a SHO Lamb wave resonator operating in the ultra-high frequency (UHF) band.

\begin{tabular}{|c|c|c|c|c|c|c|c|c|c|c|}
\hline Mode & $\begin{array}{l}\text { Orientation to } \\
+\mathrm{Y} \text { axis }\end{array}$ & $\begin{array}{l}\text { Plate Width } \\
(\mu \mathrm{m})\end{array}$ & $\begin{array}{c}\text { Aperture } \\
(\mu \mathrm{m})\end{array}$ & $\begin{array}{l}\text { Freq. } \\
\text { (MHz) }\end{array}$ & $\begin{array}{l}\mathbf{R}_{\mathbf{X}} \\
(\mathbf{\Omega})\end{array}$ & $\begin{array}{c}\mathrm{C}_{\mathrm{S}} \\
\text { (fF) }\end{array}$ & $\begin{array}{c}\text { Measured } \\
\mathbf{k}_{\text {eff }}^{2}[2,3](\%)\end{array}$ & $\begin{array}{c}\text { Measured } k_{t}^{2} \\
{[1](\%)}\end{array}$ & $\mathbf{Q}$ & $\begin{array}{c}\text { FOM } \\
k_{\text {eff }}^{2} Q /\left(1-k_{\text {eff }}{ }^{2}\right)\end{array}$ \\
\hline
\end{tabular}

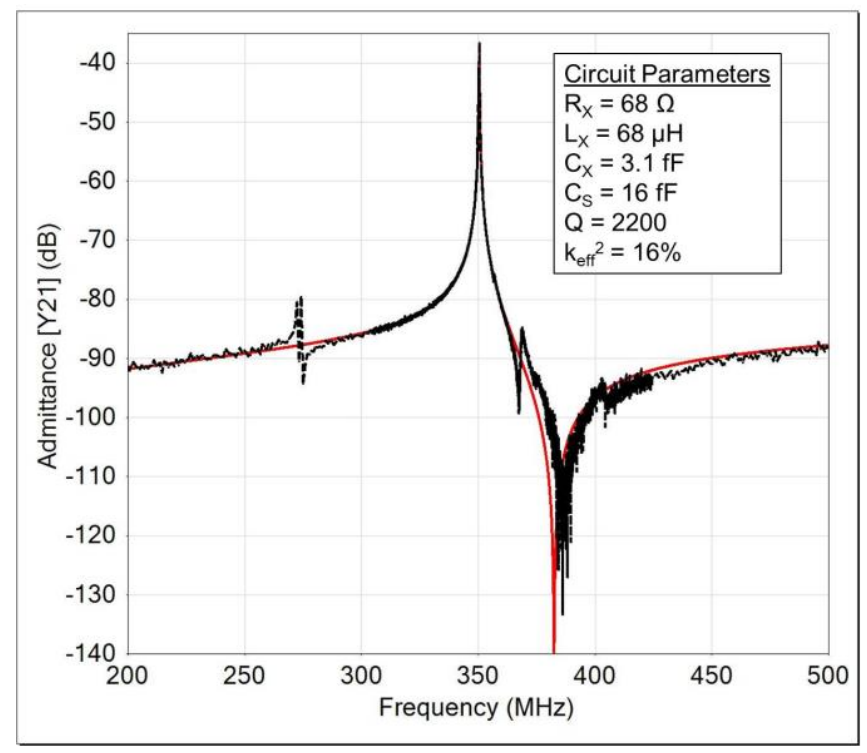

Figure 8: Experimental (black) and equivalent circuit model (red) admittance for a SHO Lamb wave resonator with a plate width of $4.4 \mu \mathrm{m}$.

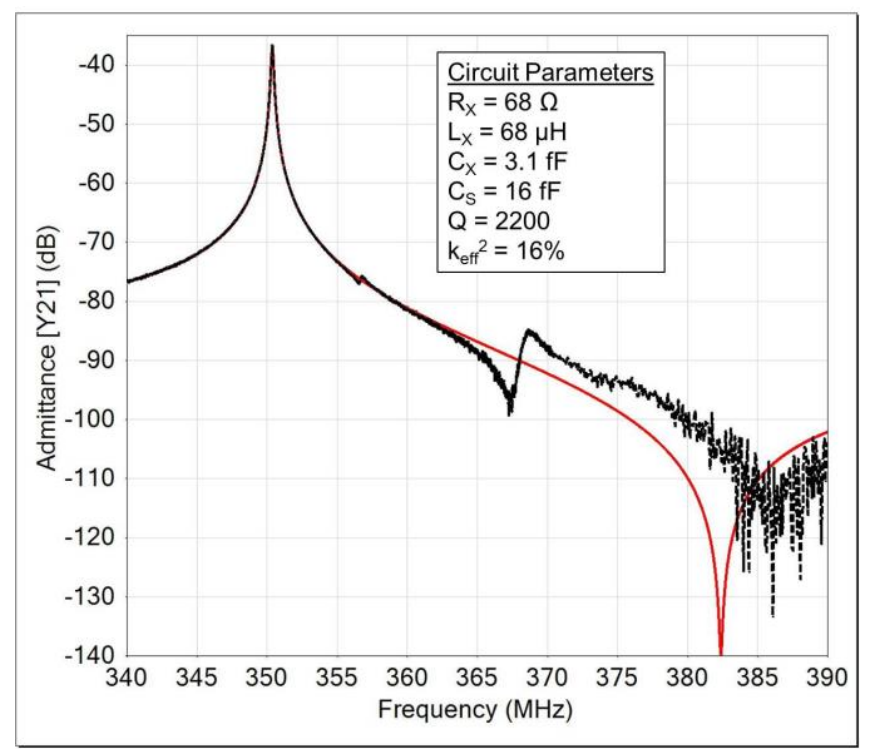

Figure 9: Experimental (black) and equivalent circuit model (red) admittance for a SHO Lamb wave resonator with a plate width of $4.4 \mu \mathrm{m}$.

\section{CONCLUSIONS}

We have studied, theoretically and experimentally, fundamental mode $\mathrm{SO}$ and $\mathrm{SHO}$ Lamb wave resonators realized in thin plates of $\mathrm{LiNbO}_{3}$. The devices are fabricated using a newly developed process that allows the formation of a damaged $\mathrm{LiNbO}_{3}$ sacrificial layer using Helium ion implantation. This damaged $\mathrm{LiNbO}_{3}$ is subsequently etched in a HF acid based wet release. The plate width, which determines the resonant frequency, is 20 $\mu \mathrm{m}$ wide for both the S0 and SH0 mode resonators and the thickness-to-wavelength ratio for both types of resonators is 0.04 , near the optimum value to maximize piezoelectric coupling found in [4]. The acoustic wave propagation is rotated $30^{\circ}$ (S0) and $170^{\circ}$ (SH0) to the $+y$-axis for the resonators, also an optimum condition to maximize piezoelectric coupling reported in [4]. We find that the SHO mode microresonators consistently exhibit higher effective piezoelectric coupling, quality factor and figure-of-merit when compared to identically designed S0 mode structures. The properties of the SH0 mode resonators are less sensitive to the device aperture and the SH0 mode resonators exhibit fewer spurious responses. While the initial study was performed on $\mathrm{LiNbO}_{3}$ microresonators operating at 97 (SH0) and 160 (S0) MHz, a SH0 mode microresonator in a $4.4 \mu \mathrm{m}$ wide $\mathrm{LiNbO}_{3}$ plate has been demonstrated at $350 \mathrm{MHz}$. The $k_{\text {eff }}^{2}=16 \%, Q=2200$ and $M$ $=420$ for the $350 \mathrm{MHz}$ microresonator are among the highest demonstrated for this new class of resonant micro-devices.

\section{ACKNOWLEDGEMENTS}

This project was partially supported by the Laboratory Directed Research and Development (LDRD) Program at Sandia National Laboratories. Sandia National Laboratories is a multiprogram laboratory managed and operated by Sandia Corporation, a wholly owned subsidiary of Lockheed Martin Corporation, for the U.S. Department of Energy's National Nuclear Security Administration under contract DE-AC04-94AL85000.

\section{REFERENCES}

[1] S. Gong and G. Piazza, "Design and analysis of lithiumniobate-based high electromechanical coupling RF-MEMS resonators for wideband filtering", IEEE Trans. on Microwave Theory and Tech., 61, 1 (2013).

[2] R.R. Wang, S.A. Bhave, and K. Bhattacharjee, "Thin-film high $\mathrm{k}_{\mathrm{t}}^{2} \mathrm{Q}$, multi-frequency lithium niobate resonators", Technical Digest of the IEEE International Conference on Micro Electro Mechanical Systems, (2013), pp. 165-168.

[3] R.H. Olsson III et al., "A high electromechanical coupling coefficient SHO Lamb wave lithium niobate micromechanical resonator and a method for fabrication", Sensors and Actuators A 209 (2014) 183-190.

[4] I.E. Kuznetsova et al., "Investigation of acoustic waves in thin plates of lithium niobate and lithium tantalite", IEEE Trans. on Ultrasonics, Ferroelectrics and Frequency Cntrl., 48, 1 (2001).

\section{CONTACT}

*R.H. Olsson III, tel: +1-505-284-6375; rholsso@ sandia.gov 